\title{
Growth Monitoring With Submonolayer Sensitivity Via Real-Time Thermal-Conductance Measurements
}

\author{
P. Ferrando-Villalba, ${ }^{1}$ D. Takegami, ${ }^{1}$ Ll. Abad,${ }^{2}$ J. Ràfols-Ribé, ${ }^{1}$ A. Lopeandia,,${ }^{1, *}$ G. Garcia,,${ }^{1}$ and \\ J. Rodriguez-Viejo ${ }^{1}$ \\ ${ }^{1}$ Grup de Nanomaterials i Microsistemes, Dep. Física, Universitat Autònoma de Barcelona, \\ 08193 Bellaterra, Spain \\ ${ }^{2}$ Institut de Microelectrònica de Barcelona-Centro Nacional de Microelectrònica - CSIC, \\ Cerdanyola del Vallès, 08193, Spain
}

(Received 20 March 2019; published 3 July 2019)

\begin{abstract}
Growth monitoring during the early stages of vapor deposition is of prime importance to understand the growth process, the microstructure, and thus the overall layer properties. We demonstrate that phonons can be used as an extremely sensitive probe to monitor the real-time evolution of film microstructure during growth, from incipient clustering to continuous film formation. For that purpose, a silicon nitride membrane-based sensor is fabricated to measure the in-plane thermal conductivity of thin film (conductive or nonconductive) samples. Operating with the $3 \omega$-Völklein method at low frequencies, the sensor shows an exceptional resolution down to $\Delta(\kappa \cdot t)=0.065 \mathrm{~W} / \mathrm{m} \mathrm{K} \mathrm{nm}$, enabling accurate measurements even in poor conductive samples. Validation of the sensor performance is done by growth characterization of organic and metallic thin films to tackle the low to high thermal-conductivity range. In both cases, at early stages of growth, the extreme sensitivity of the technique has revealed an initial reduction of the effective thermal conductance of the supporting amorphous membrane, $K$, related to the surface phonon scattering enhanced by the incipient nanoclusters formation. As cluster coalescence advances, $K$ reaches a minimum to rise up upon the percolation threshold. Subsequent island percolation produces a sharp increase of the conductance and once the surface coverage is completed $K$ increases linearly with thickness. The thermal conductivity of the deposited films is also obtained from the slope of $K$ with thickness.
\end{abstract}

DOI: 10.1103/PhysRevApplied.12.014007

\section{INTRODUCTION}

Monitoring the first stages of thin-film growth is of key importance to understand and thus tune the properties of the grown layers. Critical microstructure features, such as grain size, morphology, crystal orientation, nature of grain boundaries, and surface morphology are defined during the early growth process. Real-time measurements have proven their potentiality to understand the growth dynamics, either for thin films or nanoparticles deposited on surfaces. Actually, in situ diagnostics during growth with monolayer sensitivity have already been performed by a variety of techniques, such as wafer curvature measurements mapping the stress evolution [1], ellipsometry [2], x-ray reflectivity [3,4], and resistance-based measurements. Low or medium energy electron diffraction

\footnotetext{
*aitor.lopeandia@uab.cat
}

Published by the American Physical Society under the terms of the Creative Commons Attribution 4.0 International license. Further distribution of this work must maintain attribution to the author(s) and the published article's title, journal citation, and DOI.
(LEED, MEED) are also reliable tools to monitor twodimensional (2D) ordering during epitaxial growth [5,6]. Among all, electrical measurements are very powerful since (i) the electrical resistance in metallic thin films [7] may vary orders of magnitude above the percolation threshold [8] and (ii) drastic electrical conductivity changes during the initial growth stages can identify phase transformations [9], such as amorphous-to-crystal transition in Mo films [10]. Unfortunately, although simple and accessible, this approach is limited to metallic or highly conductive layers, precluding the analysis of organic or insulating materials. In contrast, phonons are a more generic probe, extremely sensitive to film structure thanks to their larger mean free path compared to electrons. However, real-time thermal conductance measurements during film growth are much scarcer, mainly due to the technical challenges associated with them.

Additionally, the potential application of nanomaterials, thin films, and nanostructures in heat management and efficient thermoelectric devices has boosted the necessity to perform accurate thermal-conductivity measurements at the nanoscale. In particular, phonon engineering in low-dimensional materials has appeared as the most 
effective approach to enhance the thermoelectric figure of merit [11-13] through the reduction of thermal conductivity. The implementation of alternative nanomaterials designs has encouraged the development of different sensors and methodologies, enabling accurate determination of thermal conductivity in low-dimensional architectures. Whether based on optical [14] or electrical [15] signals, these alternative thermal sensors and the associated methodologies have allowed in-plane [16,17] and outof-plane $[18,19]$ thermal-conductivity measurements of nanowires and thin films with outstanding nanometer spatial resolution [20,21]. A remarkable contribution to the field was achieved by Völklein et al. in 1990 when they developed a suspended membrane-based sensor using a long and thin Pt electrical transductor operated in dc to measure in-plane thermal conductivity of thin films [17]. More recently, Sikora et al. went a step further in improving this technology by combining the Völklein method with the ac $3 \omega$-method, reaching exceptional thermalconductance sensitivity, $\Delta K / K \cong 10^{-3}[22,23]$.

In parallel, ex situ thermal probe studies performed on thin films $[24,25]$ have shown that the thermal conductivity of a grown material is conditioned by the thermal-conductance loss of the substrate induced both by interfacial scattering of phonons in the in-plane measurements [24] and by thermal boundary resistance in the out-of-plane measurements [25]. Accordingly, the thermal conductivity of the thin layer cannot be simply calculated via the differential measurement of the thermal conductance of the whole sample (film + membrane/substrate) and a reference (only membrane/substrate), but must be calculated using a set of thermal-conductance measurements performed at different film thicknesses. To date, most of these measurements have only been performed $e x$ situ, evaluating the temperature dependence of the thermal conductivity $k$ for each singular thickness. To our knowledge, real-time studies during growth at early stages taking into account the impact of the microstructure on phonon scattering have not been previously reported. It is worth noting that although Völklein and Starz [26] already demonstrated in 1997 that a Völklein-like sensor operating in dc mode could perform in situ measurement of thermal conductance in thin films, those measurements were limited to metallic films thicker than $1 \mu \mathrm{m}$, contrary to the sensor presented here.

In this paper, we present (i) an improved $3 \omega$-Völklein technique allowing highly sensitive conductance measurements $\left(\Delta K / K<10^{-3}\right)$, (ii) the sensor optimization through FEM (Finite Element Modeling) thermal analysis, (iii) an innovative study of the thermal evolution of the sensor during growth, and (iv) real-time measurements of the thermal conductance during growth of both highly insulating and conductive (electrically and thermally) materials with submonolayer sensitivity. Proof-of-concept is achieved by analyzing two different materials: a metal, such as In (indium), and an organic conductor, N,N'-Bis (3-methylphenyl)-N, $\mathrm{N}^{\prime}$-diphenylbenzidine (TPD), often used as a hole injector in organic light-emitting diode (OLEDs). Complementary characterization techniques (SEM, AFM, and electrical measurements) are used to correlate conductance features with sample morphology throughout the growth.

\section{EXPERIMENTAL SECTION}

\section{A. Sensor design and simulation}

The sensor developed here consists of a long and thin Pt line deposited on a suspended $\operatorname{SiN}_{x}$ membrane and connected in a four-wire configuration [Figs. 1(a) and 1(b)] along with an external Pt line. The thermal conductance of the whole membrane is determined with the centered Pt line (normal operation), while both lines, centered and external, are needed to exclusively measure the thermal conductance of the membrane volume portion between them. See Fig. S1 in the Supplemental Material [27] for details of the sensor microfabrication.

In normal operation, the thermal conductance is calculated by using the one-dimensional (1D) Fourier law, assuming an ideal transversal heat flow from the central Pt line to the substrate toward the Si frame, which yields

$$
K=k_{\operatorname{SiN}_{x}} t_{\operatorname{SiN}_{x}}\left(2 \frac{L}{\lambda}\right)
$$

where $t_{\mathrm{SiN}_{x}}$ and $k_{\mathrm{SiN}_{x}}$ are the thickness and the thermal conductivity of the silicon nitride, respectively, $L$ is the length of the Pt strip between the voltage probes, and $\lambda$ is the transversal distance between the central Pt strip (linewidth $b=5 \mu \mathrm{m})$ and the Si bulky substrate.

Using the classical diffusive Fourier equations for heat transport and FEM with COMSOL software, we model the heat transport in several sensor geometries. Considering the Joule heating produced by a dc current flowing through the Pt central strip, the simulated steady-state temperature profiles are obtained. The central part of the strip always shows a flatter temperature profile than its borders, information that is used to determine the location of voltage probes in the central strip to ensure that the sensing area is limited taken with maximizing the temperature homogeneity.

The optimum sensor (taking into account the resolution limits of the available photolithographic system and the structural stability of the $\operatorname{SiN}_{x}$ membrane) consists of a long and narrow $\mathrm{SiN}_{x}$ membrane $(3 \mathrm{~mm} \times 250 \mu \mathrm{m} \times$ $180 \mathrm{~nm}$ ) that supports the two Pt sensing strips $(3 \mathrm{~mm} \times 5 \mu \mathrm{m} \times 100 \mathrm{~nm}$ ) with voltage probes separated by $2 \mathrm{~mm}$. The electrical lines of the voltage probes enter the membrane following in close proximity to the central $\mathrm{Pt}$ strip, minimizing heat leakage and thus temperature drop along the strip. The simulated profile of the longitudinal 

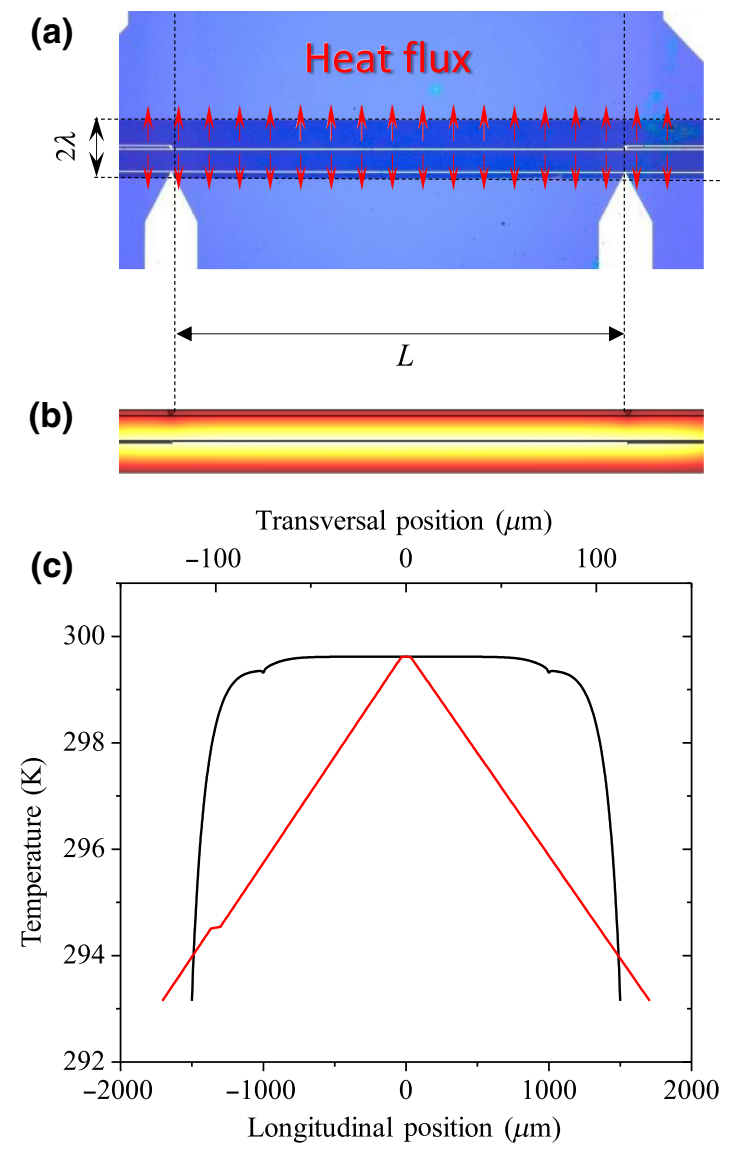

FIG. 1. (a) Optical micrograph of the sensor consisting on a suspended rectangular membrane (with a total width of $2 \lambda$ ) a longitudinal strip Pt heater placed on the center with a distance $\mathrm{L}$ between the four wire electrical probes. Red arrows indicated the ideal heat flow directions. (b) Surface temperature distribution of the sensor (brighter is hotter) in a FEM dc study. (c) Longitudinal and transversal temperature distributions of the sensor in a FEM dc study.

temperature [black line in Fig. 1(c)] shows a large and flat central plateau where the inhomogeneity is lower than 3\% of the total temperature rise. We notice two tiny temperature depressions at $\pm 1000 \mu \mathrm{m}$, coinciding with the voltage probe locations. The transversal profile exhibits a constant slope only perturbed by minimal flat segments corresponding with the position of the Pt strips. With this sensor, the thermal conductivity of the $\mathrm{SiN}_{x}$ layer can be measured with an accuracy of $0.05 \%$ in the absence of other uncertainty sources. See the Supplemental Material [27] for more information about the modeling.

\section{B. Thermal analysis during film deposition}

Based on Sikora et al. developments [22,23], we deduce a mathematical expression to extract the thermal conductivity from the measured thermal conductance at different thicknesses. When the central Pt line is fed with an oscillating current $I=I_{0} \sin \omega t$, the amplitude of the induced temperature oscillations can be calculated with the 1D heat equation (assuming that the sensor is infinitely long), which, after some approximations (see the Supplemental Material [27] for details of mathematical derivation), yields

$$
\Delta T_{2 \omega}=\frac{P_{0}}{K \sqrt{1+\omega^{2}\left(4 \tau^{2}+\frac{2 \lambda^{4}}{3 D^{2}}+\frac{4 \tau \lambda^{2}}{3 D}\right)}},
$$

where $P_{0}=I_{o}^{2} R / 2, D=k_{\mathrm{SiN}_{x}} /\left(\rho_{\mathrm{SiN}_{x}} c_{\mathrm{SiN}_{x}}\right), \quad \tau=C_{p} / K$, and $C_{p}=b L\left(\rho_{\mathrm{SiN}_{x}} c_{\mathrm{SiN}_{x}} t_{\mathrm{SiN}_{x}}+\rho_{\mathrm{Pt}} c_{\mathrm{Pt}} t_{\mathrm{Pt}}\right)$. Here, $\rho_{\mathrm{SiN}_{x}, \mathrm{Pt}}$ is the density of the $\mathrm{SiN}_{x}$ membrane and the Pt strip, $c_{\mathrm{SiN}_{x}, \mathrm{Pt}}$ is their specific heat capacity, $C_{p}$ is the heat capacity of the strip and the portion of $\operatorname{SiN}_{x}$ beneath it, $\tau$ is the thermal time constant of the sensor, $D$ is the thermal diffusivity of $\mathrm{SiN}_{x}, R$ is the Pt strip electrical resistance between the voltage probes, and $I_{0}$ is the current amplitude. From Eq. (2), the apparent thermal conductance can be calculated as $K_{2 \omega}=P_{0} / \Delta T_{2 \omega}$, which resembles $K$ at low frequencies.

When a thin-film sample grows on the $\mathrm{SiN}_{x}$ membrane, the parameters in Eq. (2) may vary as $k, c$, and $\rho$ will no longer correspond solely to the $\operatorname{SiN}_{x}$ membrane, but to the combination of the deposited film (from now on called "sample") and the substrate membrane. In this case, an effective value for these magnitudes can be calculated (assuming a vertical growth of the film) by pondering the different values as a function of the film thickness, $t_{\mathrm{smp}}$

$$
\begin{aligned}
& k_{\mathrm{eff}}=\frac{k_{\mathrm{SiN}_{x}} \cdot t_{\mathrm{SiN}_{x}}+k_{\mathrm{smp}} \cdot t_{\mathrm{smp}}}{t_{\mathrm{SiN}_{x}}+t_{\mathrm{smp}}}, \\
& c_{\mathrm{eff}}=\frac{c_{\mathrm{SiN}_{x}} \cdot t_{\mathrm{SiN}_{x}}+c_{\mathrm{smp}} \cdot t_{\mathrm{smp}}}{t_{\mathrm{SiN}_{x}}+t_{\mathrm{smp}}}, \\
& \rho_{\mathrm{eff}}=\frac{\rho_{\mathrm{SiN}_{x}} \cdot t_{\mathrm{SiN}_{x}}+\rho_{\mathrm{smp}} \cdot t_{\mathrm{smp}}}{t_{\mathrm{SiN}_{x}}+t_{\mathrm{smp}}}, \\
& D_{\mathrm{eff}}=\frac{k_{\mathrm{eff}}}{\rho_{\mathrm{eff}} \cdot c_{\mathrm{eff}}} .
\end{aligned}
$$

Here, $k_{\mathrm{smp}}, c_{\mathrm{smp}}$, and $\rho_{\mathrm{smp}}$ are the sample thermal conductivity, specific heat capacity, and density. In the same way, the extrinsic values $K$ and $C_{p}$ will vary as well as the thermal time constant $\tau$ as

$$
\begin{aligned}
K\left(t_{\mathrm{smp}}\right)= & \left(k_{\mathrm{SiN}_{x}} \cdot t_{\mathrm{SiN}_{x}}+k_{\mathrm{smp}} \cdot t_{\mathrm{smp}}\right)\left(2 \frac{L}{\lambda}\right), \\
C_{p}\left(t_{\mathrm{smp}}\right)= & b L\left(\rho_{\mathrm{SiN}_{x}} \cdot c_{\mathrm{SiN}_{x}} \cdot t_{\mathrm{SiN}_{x}}+\rho_{\mathrm{Pt}} \cdot c_{\mathrm{Pt}} \cdot t_{\mathrm{Pt}}\right. \\
& \left.+\rho_{\mathrm{smp}} \cdot c_{\mathrm{smp}} \cdot t_{\mathrm{smp}}\right), \\
\tau^{\prime}= & \frac{C_{p}\left(t_{\mathrm{smp}}\right)}{K\left(t_{\mathrm{smp}}\right)} .
\end{aligned}
$$

If the measuring frequency is low ( $\left.\omega \ll 3 D_{\text {eff }} / 8 \lambda^{2}\right)$, then the measured $K_{2 \omega}$ resembles $K$ and the derivative of 
Eq. (7) can be used to calculate the thermal conductivity of the sample film by measuring in real time the thermal conductance during growth $\left[K_{2 \omega}\left(t_{\mathrm{smp}}\right)\right]$, as shown in Eq. (10).

$$
\frac{d K}{d t_{\mathrm{smp}}}=k_{\mathrm{smp}}\left(2 \frac{L}{\lambda}\right) \rightarrow k_{\mathrm{smp}}=\frac{\frac{d K}{d t_{\mathrm{smp}}}}{2 \frac{L}{\lambda}}
$$

However, if a higher current angular frequency $\omega$ is used, $k_{\text {smp }}$ can be extracted by fitting the measured $K_{2 \omega}$ with Eq. (11) using the thickness-dependent parameters (Eqs. (6)-(9))

$$
K_{2 \omega}\left(t_{\mathrm{smp}}\right)=K\left(t_{\mathrm{smp}}\right) \sqrt{1+\omega^{2}\left(4 \tau^{\prime 2}+\frac{2 \lambda^{4}}{3 D_{\mathrm{eff}}^{2}}+\frac{4 \tau^{\prime} \lambda^{2}}{3 D_{\mathrm{eff}}}\right)} .
$$

In Fig. 2(a) the calculated $K_{2 \omega}$ is plotted for current frequencies of $0 \mathrm{~Hz}(\mathrm{dc}), 1$ and $3 \mathrm{~Hz}$ (the parameters used are listed in the figure caption). If the conductance is monitored with a current at $1 \mathrm{~Hz}$, the apparent thermal conductance $K_{2 \omega}$ is very similar to $K$ throughout the deposition. However, at $3 \mathrm{~Hz}$, there is an evident difference in the slope of the curves: although the absolute value of the apparent thermal conductance only varies from $97 \%$ to $93 \%$ of $K$ [Fig. 2(b))], the slope of $K_{2 \omega}$ is up to $40 \%$ higher than the one of $K$ [Fig. 2(a)]. Generally, measuring with higher frequencies increases the dependence of $K_{2 \omega}$ with intrinsic properties of the sample that may not be well known (like $c_{\text {smp }}$ or $\rho_{\text {smp }}$ ), hindering the determination of $k_{\text {smp }}$.

\section{Experimental setup}

The sensor is introduced in a high vacuum chamber equipped with an effusion cell that enables a good control of the evaporation rate to deposit the sample at the backside of the suspended membrane. A previously calibrated quartz crystal microbalance (QCM) is located near the sensor to monitor the layer growth rate with a precision of $0.01 \AA / \mathrm{s}$. A set of shutters permits to shadow the deposition selectively in the device, in the QCM, or in both.

The temperature of the sample is controlled with a custom-made proportional-integral-derivative (PID) controller system that reads the temperature of a Pt100 and provides heat through a Kapton heater, yielding a temperature control with fluctuations smaller than $0.003 \mathrm{~K}$ from $77 \mathrm{~K}$ up to $400 \mathrm{~K}$.

The experiment is performed by feeding two sensors (sample and reference) with a current wave of a given amplitude and frequency, generating a voltage drop in each sensor. The voltage signals from both sensors, as well as the differential voltage between them, are subtracted using the low-noise amplifiers with gains $G_{\text {smp }}, G_{\text {ref }}$, and $G_{\text {diff, }}$, respectively. The reference sensor is a twin (equal to the (a)
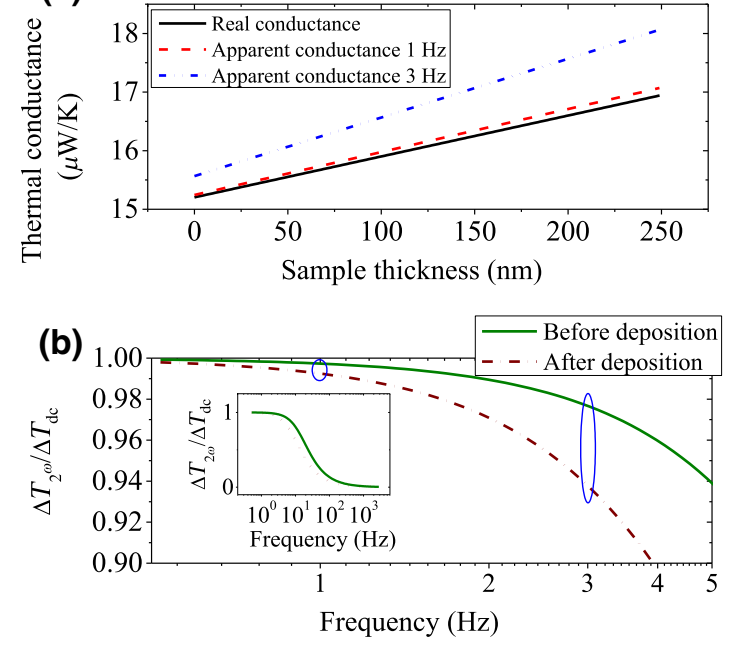

FIG. 2. (a) Calculated real thermal conductance (black) and apparent thermal conductance modeled at $1 \mathrm{~Hz}$ (red) and $3 \mathrm{~Hz}$ (blue) during the deposition of a layer. (b) Calculated frequency dependence of the temperature oscillations in the sensor before and after depositing a $250-\mathrm{nm}$ film (the inset is a zoom out).The material properties used in both plots are the following: $k_{\mathrm{SiN}_{x}}=2.65 \mathrm{~W} \mathrm{~m}^{-1} \mathrm{~K}^{-1}, c_{\mathrm{SiN}_{x}}=0.7 \mathrm{~J} \mathrm{~K}^{-1} \mathrm{~kg}^{-1}, \rho_{\mathrm{SiN}_{x}}=$ $3.18 \mathrm{~g} / \mathrm{cm}^{3}, k_{\mathrm{Pt}}=33 \mathrm{~W} \mathrm{~m}^{-1} \mathrm{~K}^{-1}, c_{\mathrm{Pt}}=0.133 \mathrm{~J} \mathrm{~K}^{-1} \mathrm{~kg}^{-1}, \rho_{\mathrm{Pt}}=$ $21.45 \mathrm{~g} / \mathrm{cm}^{3}, k_{\mathrm{smp}}=0.21 \mathrm{~W} \mathrm{~m}^{-1} \mathrm{~K}^{-1}, c_{\mathrm{smp}}=1.05 \mathrm{~J} \mathrm{~K}^{-1} \mathrm{~kg}^{-1}$, and $\rho_{\text {smp }}=1.08 \mathrm{~g} / \mathrm{cm}^{3}$. Structural parameters are $t_{\mathrm{SiN}_{x}}=$ $180 \mathrm{~nm}, t_{\mathrm{Pt}}=110 \mathrm{~nm}, \lambda=123 \mu \mathrm{m}, \quad b=5 \mu \mathrm{m}$, and $L=$ $2000 \mu \mathrm{m}$.

sample one) nonsuspended sensor, which produces no selfheating and is used to subtract the $1 \omega$ component of the sample sensor. Thus, the $3 \omega$ component of the differential voltage is only produced in the sample sensor and owing to the cancellation of the $1 \omega$ voltage, it could be amplified with a gain $G_{\text {diff }}=75$. The main benefit of using a twin sensor (instead of a variable resistance) as a reference for the differential measurement is that if the temperature of the sample holder varies, the resistance of both sensors will change hand-in-hand, making it unnecessary to build a control system for the cancellation of the $1 \omega$ voltage. The exact electronics used in the measurement of the different voltage signals are detailed in Fig. S4 of the Supplemental Material [27].

From the measured voltage signals, the resistance $R_{\mathrm{smp}}$ and the temperature oscillations $\Delta T_{2 \omega}$ can be calculated as

$$
\begin{aligned}
R_{\mathrm{smp}} & =\frac{V_{1 \omega}}{I_{0} \cdot G_{\mathrm{smp}}}, \\
\Delta T_{2 \omega} & =\frac{2 V_{3 \omega}}{I_{0} \frac{d R_{\mathrm{smp}}}{d T} \cdot G_{\mathrm{diff}} \cdot G_{\mathrm{smp}}},
\end{aligned}
$$

where $V_{1 \omega}$ and $V_{3 \omega}$ are the $1 \omega$ and $3 \omega$ voltage components measured in the sample sensor and $d R_{\text {smp }} / d T$ is the slope of the sample resistance as a function of the temperature. 


\section{RESULTS}

\section{A. Sensor performance test}

Initially, the self-heating of the Pt sensor is determined by measuring both the $3 \omega$ voltage and the variation of the $1 \omega$ voltage with $\omega=2 \pi \mathrm{rad} / \mathrm{s}$. Since the frequency is very low, both signals yield identical self-heating, but as can be seen in Fig. 3(a), the self-heating calculated with the $3 \omega$ voltage $\left(\Delta T_{2 \omega}\right)$ is less noisy than the one calculated with the $1 \omega$ voltage $\left(\Delta T_{\mathrm{dc}}\right)$. Also, the slope of $\log \Delta T_{2 \omega}$ vs $\log I_{0}$ has a value very close to 2 , demonstrating that the self-heating depends on the square of the current.

$\Delta T_{2 \omega}$ has been measured in a wide frequency range $(1-2000 \mathrm{~Hz})$ and compared with finite element simulations in a time-dependent study, as can be seen in Fig. 3(b). The high coincidence between both datasets suggests that the behavior of the sensor is purely driven by heat transport physics. This is an important difference from the device presented by Sikora et al. [22], where the use of a $\mathrm{NbN}$ strip sensor allowed measurements at very low temperatures, but produced non-negligible electrical effects due to the high electrical impedance of that material.

Finally, as shown in Fig. 3(c), the uncertainty of the conductance measurement is determined at two different current intensities, 300 and $500 \mu \mathrm{A}$, which generate temperature amplitudes of about 2 and $6 \mathrm{~K}$ and produced
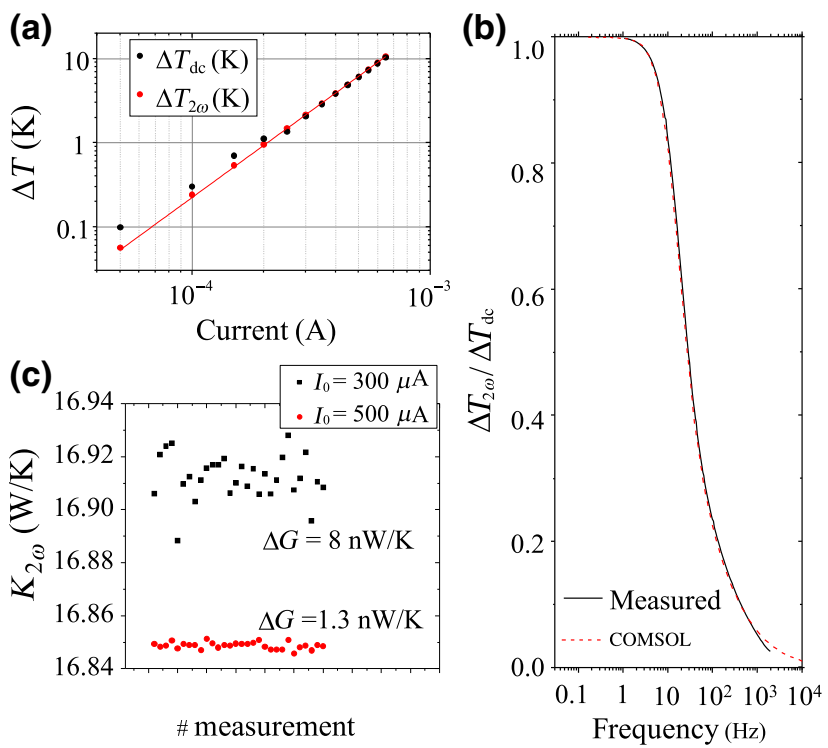

FIG. 3. (a) Self-heating of the sensor measured with the $3 \omega$ and $1 \omega$ voltages $(\omega=1 \mathrm{~Hz})$. (b) Temperature oscillations as a function of the frequency. The measured values are compared with the simulated ones using the COMSOL model. (c) Measurement of the uncertainty in the thermal conductance. $\Delta K$ refers to the standard deviation of the data. Red circles and black squares correspond to data obtained with input currents of 500 and $300 \mu \mathrm{A}$, respectively, with $\omega=1 \mathrm{~Hz}$. a standard deviation of data equal to 8 and $1.3 \mathrm{nW} / \mathrm{K}$, respectively. This accuracy in the thermal conductance provides an extremely high resolution in the product of the thermal conductivity and the thickness, $\Delta(k \cdot t)=$ $0.065 \mathrm{~W} / \mathrm{m} \mathrm{K} \mathrm{nm}$. Although there is a decrease in the data dispersion for $500 \mu \mathrm{A}$, there is also a noticeable reduction in the average value of the thermal conductance from 16.91 to $16.85 \mu \mathrm{W} / \mathrm{K}$ that may be justified by a small reduction of the sensor $d R / d T$ due to the higher average heater temperature.

The thermal conductivity of the $\mathrm{SiN}_{x}$ membrane is evaluated for temperatures between 80 and $230 \mathrm{~K}$, as shown in Fig. 4 (black squares). The results are very similar to the values found in the out-of-plane direction using the $3 \omega$ method on a similar $\mathrm{SiN}_{x}$ membrane (red circles in Fig. 4). The latter values are obtained through a differential measurement of two samples with thicknesses of 180 and $450 \mathrm{~nm}$, granting that the thermal boundary resistances between the film and the substrate are cancelled. Thus, the similarity of both the in-plane and the out-of-plane values confirm that there are not substantial phonon size effects, or anisotropy, in our layers. In both cases, the variation of $k$ with temperature shows a similar tendency to data presented by Sikora et al. [22,23] (continuous line in Fig. 4). The discrepancy in the absolute values may account for density or stoichiometry variations related to the different growth film characteristics in each work. Huge differences in $k$ variation with temperature are observed when comparing our data with the work of Sultan et al. [28] (blue triangles in Fig. 4). We suggest, as will be discussed later, that this difference is probably related to fact that they use a nanocrystalline membrane.

The measurements performed in this work have an extremely low variability owing to the high sensitivity of the method. The main source of uncertainty in the in-plane

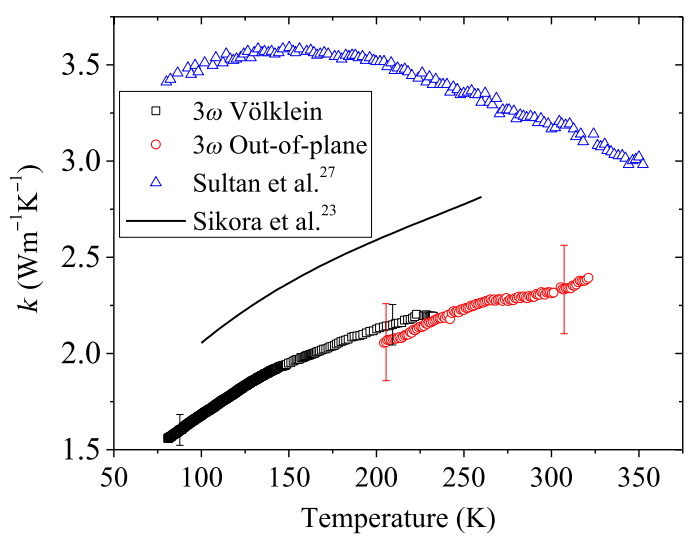

FIG. 4. Thermal conductivity values measured at different temperatures with the $3 \omega$-Völklein method (in-plane direction) and the $3 \omega$ method (out-of-plane direction). Data from Sultan et al. [28] and Sikora et al. [23] are also included for comparison. 
measurement is the precise determination of $\mathrm{SiN}_{x}$ membrane thickness, which is measured throughout the wafer with a contactless optical profilometer, yielding a standard deviation of $1 \%$ of the $180-\mathrm{nm}$ averaged thickness. However, wet etchings at the final steps of microfabrication process could slightly reduce this thickness by up to $10 \mathrm{~nm}$, inevitably increasing the uncertainty up to $5 \%$.

\section{B. Real-time measurement of thin-film growth: sensor proof of concept \\ 1. Organic thin-film layers}

We initially measure the thermal conductance of the membrane as a function of the TPD thickness deposited at $267 \pm 2 \mathrm{~K}$, as shown in Fig. 5(a). The temperature uncertainty comes from the thermal oscillations produced by the current wave of amplitude $300 \mu \mathrm{A}$. The evaporation is carried out below $10^{-7}$ mbar by heating an effusion cell up to $200^{\circ} \mathrm{C}$, which yields a stable deposition rate of $0.29 \mathrm{~nm} / \mathrm{s}$. From the slope of the curve $K_{2 \omega}\left(t_{\mathrm{smp}}\right)$ and applying Eq. (8), we calculate the thermal conductivity of the TPD layer, resulting in $k_{\mathrm{smp}}=$ $(0.153 \pm 0.001) \mathrm{W} \mathrm{m}^{-1} \mathrm{~K}^{-1}$. However, if the thermal conductivity is calculated by fitting Eq. (11), we obtain $k_{\mathrm{smp}}=$ $(0.145 \pm 0.001) \mathrm{W} \mathrm{m}^{-1} \mathrm{~K}^{-1}$. As discussed previously, both results are comparable due to the low frequency used in the measurement. The low value of the thermal conductivity is an indication of the glassy character of the TPD layers as confirmed by calorimetric measurements showing a clear signature of the glass transition temperature, as can be seen in Fig. S7 in the Supplemental Material [27].

As clearly seen in Fig. 5(a), at the early stages of the deposition, that is, very low thicknesses, we observe a decrease in the thermal conductance of around $1.2 \%$ of its initial value, while afterward, $K_{2 \omega}$ roughly increases linearly with thickness. In this particular measurement, film growth is stopped at $340 \mathrm{~nm}$, yielding a constant value of the thermal conductance after this particular point.

Figures 5(b) and 5(c) show in more detail the initial stages of the evolution of $K_{2 \omega}$ vs thickness for two TPD films deposited at 267 and $304 \mathrm{~K}$, both with a low growth rate of $0.02 \mathrm{~nm} / \mathrm{s}$. In both cases, we identify four different regions where thermal conductance follows different trends with different thicknesses. In region I, the overall conductance decreases abruptly following an exponential behavior, while it decreases slowly in region II until a minimum point is reached. Region III covers the thickness range where $K_{2 \omega}$ increases slowly up to a point where a linear regime of the conductance with thickness is attained and labeled as region IV. The extent of each of these regions is represented in Figs. 5(b) and 5(c) by dashed red lines, and as is clearly seen, varies from one sample to another, confirming the importance of deposition temperature as will be discussed later.
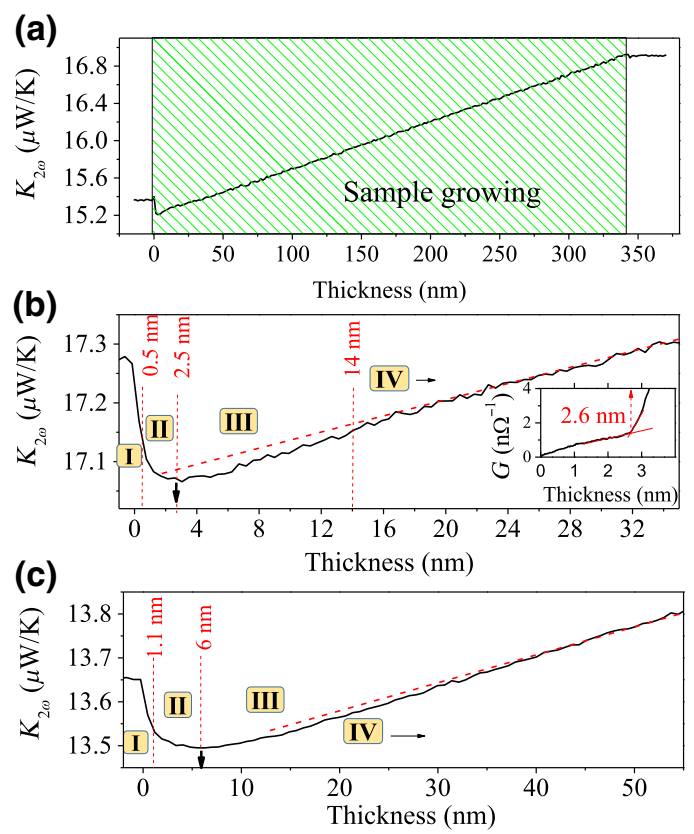

FIG. 5. Thermal conductance versus thickness during TPD growth: (a) $T_{\text {dep }}=267 \pm 2 \mathrm{~K}$. Growth rate $=0.29 \mathrm{~nm} / \mathrm{s}$. (b) $T_{\text {dep }}=267 \pm 2 \mathrm{~K}$. Growth rate $=0.02 \mathrm{~nm} / \mathrm{s}$. (c) $T_{\text {dep }}=304 \pm$ $2 \mathrm{~K}$. Growth rate $=0.02 \mathrm{~nm} / \mathrm{s}$. In graphs (b) and (c), data are box averaged with 10 points/box. The main regions of film growth are separated by dashed red lines. Region I located between $0 \mathrm{~nm}$ and the first vertical line corresponds to nucleation and isolated island formation; region II to island coalescence; region III to percolation across the layer, and region IV to vertical growth of a continuous layer. The slight difference in the initial conductance between all the graphs is mainly due to the use of different sensors and/or membranes for the experiments. The black downward arrow marks the percolation threshold (separation between regions II and III). The inset of (b) shows the abrupt variation of the electrical conductance at the percolation threshold (nominal thickness of $2.6 \mathrm{~nm}$ ) of the TPD film. This value coincides with the minimum of the thermal conductance (black arrow) in graph (b). The uncertainty of the thermal conductance measurement in this measurements is below $0.1 \%$.

To understand and interpret the variation of $K_{2 \omega}$ with thickness, we also perform ex situ analysis of the film morphology either by SEM or by AFM, summarized in Fig. S5(a) in the Supplemental Material [27]. Complementary electrical characterizations are also performed, as shown in Fig. 5(b) inset, to corroborate the hypothesis described later on. Surface morphology evolution with thickness show that TPD does not wet the $\mathrm{SiN}_{x}$ membrane surface, as it grows in 3D islands in the early growth stages. For films deposited at $267 \mathrm{~K}$ and according to the conductance evolution, island coalescence seems to happen at a nominal film thickness of $2.6 \mathrm{~nm}$, while complete surface coverage seems to take place at $14 \mathrm{~nm}$. Ex situ analysis of growth regimes is precluded by the evidence of dewetting of TPD at temperatures well below the glass transition 
temperature [29].The fast film dynamics at temperatures below the glass transition temperature makes in situ analysis an indispensable tool to gain a better understanding of film formation in molecular glasses.

Before relating the large drop observed in region I with film microstructure, we perform a few tests to rule out any potential experimental artifact that could have led to temperature variations of the sensor. As extra heat originated by condensation of molecules on the substrate is not modulated at angular frequency $\omega$ or any of its harmonics, and therefore, does not affect the $3 \omega$ component of the voltage, the decrease of conductance cannot be attributed to a sensor temperature variation due to this phenomenon. We also rule out any effect of the sample emissivity variations during TPD growth on the membrane, since a measurement at $81 \mathrm{~K}$, where radiation effects should be substantially lower, shows a similar drop in thermal conductance (measurement shown in Fig. S6 in the Supplemental Material [27]). The reduction of thermal conductance observed in the early stages of growth seems to be due to phononrelated phenomena, although covering the surface with TPD is not expected to affect the thermal conductance of the $\mathrm{SiN}_{x}$ membrane since it is already a disordered structure with an average phonon mean free path of the order of the interatomic distance. Nevertheless, a previous work by Sultan et al. $[24,28]$ already showed similar behavior $(K$ decreases at early growth stages with a subsequent increase for larger thicknesses) by performing ex situ measurements of thermal conductance versus thickness of metal and alumina deposited on $\mathrm{SiN}_{x}$. Nonetheless, our data differs from this previous work in several important aspects: (i) the magnitude of the conductance drop is much lower and (ii) our measurements are carried out in situ with a much higher conductance sensitivity, providing a much clearer signature of the various growth regimes.

Our thermal conductance initial drop, although much higher than the uncertainty of the measurement, only amounts to $1.2 \%$ compared to $5-10 \%$ in Sultan's work. Those authors estimated that $40 \%-50 \%$ of the total $K$ was due to long wavelengths with long mean free path phonons. The estimated average $\lambda$ value for these phonons was around $4.5 \mathrm{~nm}$, much higher than thermal phonons at room-temperature that have $\lambda \sim 0.2 \mathrm{~nm}$. We thus believe that the main difference between both results arises from a different microstructure of the $\mathrm{SiN}_{x}$ membrane in each case. The thermal conductivity value of our $\mathrm{SiN}_{x}$, slightly below Sultan et al. [24,28] values, combined with the temperature dependence shown in Fig. 4, clearly indicates that our nitride is fully amorphous while the one used in Sul$\tan$ et al. work was probably nanocrystalline. Therefore, a lower drop of the thermal conductance in fully amorphous nitride membrane is consistent with a scenario where the contribution of long $\lambda$ phonons is reduced with respect to previous nanocrystalline nitride membranes, even though the conductance drop due to enhanced surface scattering requires that phonons slightly larger than the average interatomic distance at room temperature be involved in heat transport along the nitride membrane. We can thus tentatively attribute the initial sharp reduction of the thermal conductance to the formation of TPD isolated clusters on top of the nitride membrane surface, modifying the interfacial phonon scattering and thus leading to a decrease of the thermal conductance.

Although we currently lack a complete understanding of the microscopic processes occurring at the interfaces, we believe that the growth of new material on top of the membrane changes the specularity of the surface, resulting in an effective increase in the phonon scattering rate. We foresee that the future use of crystalline membranes, such as single crystalline $\mathrm{Si}$, will provide a convenient platform to investigate nucleation and island growth dynamics during the early stages of film growth with even higher conductance sensitivity.

In region II, the thermal conductance still decreases but with a lower slope. We believe that in this region, clusters enlarge and start to coalesce, providing additional paths for heat transfer, which partially compensate the interface scattering until a minimum is reached at the end of the present region. As island coalescence continues and percolation builds up new channels across the layer structure (region III), providing additional heat flow paths that start to exceed the contribution of the interface scattering. The coincidence of the minimum in thermal conductance with a percolation phenomenon threshold is demonstrated in a separate experiment where electrical conductance is measured as a function of thickness [inset in Fig. 5(b)], showing a sharp variation of the slope at $2.6 \mathrm{~nm}$ due to electrical continuity through the TPD film.

As percolation persists, the thermal conductance increases, reaching a linear regime that we identify with the formation of a continuous layer with complete coverage, and marking the onset of region IV. Thus, region IV corresponds to the vertical growth of the continuous film. In this regime, the increase in thermal conductance is linear and proportional to the thickness of the growing layer. Compared to the end of region III, there is a small reduction in the slope of the conductance versus thickness since the islands are no longer forming new conductive channels.

Differences in growth dynamics with the deposition temperature are also studied, as shown in Fig. 5(c), where TPD film is deposited at $304 \mathrm{~K}$. Although the four regions are evident in both cases, remarkable differences in region limits appear with respect to the sample deposited at $267 \mathrm{~K}$ [Fig. 5(b)]. At this temperature, the percolation threshold (black downward arrows) and film continuity are 2.5 and $14 \mathrm{~nm}$, respectively. However, a sample grown at $304 \mathrm{~K}$ shows a higher percolation threshold of $6 \mathrm{~nm}$ (probably due to higher molecular mobility taking place at a higher deposition temperature), and the thickness value for film continuity is not clearly resolved from the data since the 
reduction in the slope after region III is not observed. Recent work by Fakhraai and coworkers [29] in TPD films grown at $315 \mathrm{~K}$ have shown that film continuity was reached for film thicknesses above $20 \mathrm{~nm}$, which is consistent with our results.

Although a similar behavior of $K_{2 \omega}$ is observed for both samples, final values of in-plane conductivity determined from the slope in regions IV of Figs. 5(a) and 5(c) differ considerably: $k=0.145 \mathrm{~W} \mathrm{~m}^{-1} \mathrm{~K}^{-1}$ for films deposited at $T_{\text {dep }}=267 \mathrm{~K}$ and $k=0.132 \mathrm{~W} \mathrm{~m}^{-1} \mathrm{~K}^{-1}$ for those deposited at $T_{\text {dep }}=304 \mathrm{~K}$. While the difference only amounts to $10 \%$, it is substantially larger than the measurement uncertainty. Both films are amorphous at those deposition temperatures, as confirmed by clear glass transition signatures shown in the calorimetric traces of Fig. S7 in the Supplemental Material [27]. The variations in thermal transport are related to the intrinsic characteristics of vapor-deposited glasses, in particular, density and molecular orientation, which strongly depend on the deposition temperature. As shown in a previous publication [30], the degree of molecular packing anisotropy depends on the deposition temperature and substantially influences thermal transport, while the small density variations do play a minor role. TPD films grown at $0.80 T_{g}$ have molecules with the long axis partially aligned parallel to the substrate, while those grown at $0.91 T_{g}$ are mostly randomly oriented [31]. We have recently demonstrated that heat transport along the backbone (the long axis of the molecule) is strongly preferred with respect to transport through the perpendicular direction. Therefore, the higher thermal conductivity value measured at $T_{\text {dep }}=267 \mathrm{~K}$ compared to the one obtained for samples grown at $T_{\text {dep }}=305 \mathrm{~K}$ is related to variations in the molecular orientation.

\section{Metallic thin-film layers}

To complete the proof of concept of the sensor, we also analyze growth kinetics of a metallic indium layer. Figure 6 shows the real-time in situ thermal conductance measurement during growth for two deposition temperatures, 315 and $260 \mathrm{~K}$. Conductance values [Figs. 6(a) and $6(\mathrm{~b})$ ] follow a similar pattern to the one observed in TPD films, but with much larger thickness scales. The conductance regions can be identified and discussed, as in the TPD case, in view of the microstructure evolution with thickness (see Fig. S5 in Supplemental Material [27] for a complete set of microscopy images). As shown in the inset of Fig. 6(a), the fast decrease of conductance at the very early stages of deposition is also present. In this thickness range (up to $0.65 \mathrm{~nm}$ ), tiny isolated clusters are expected to develop on the surface of the $\mathrm{SiN}_{x}$ membrane.
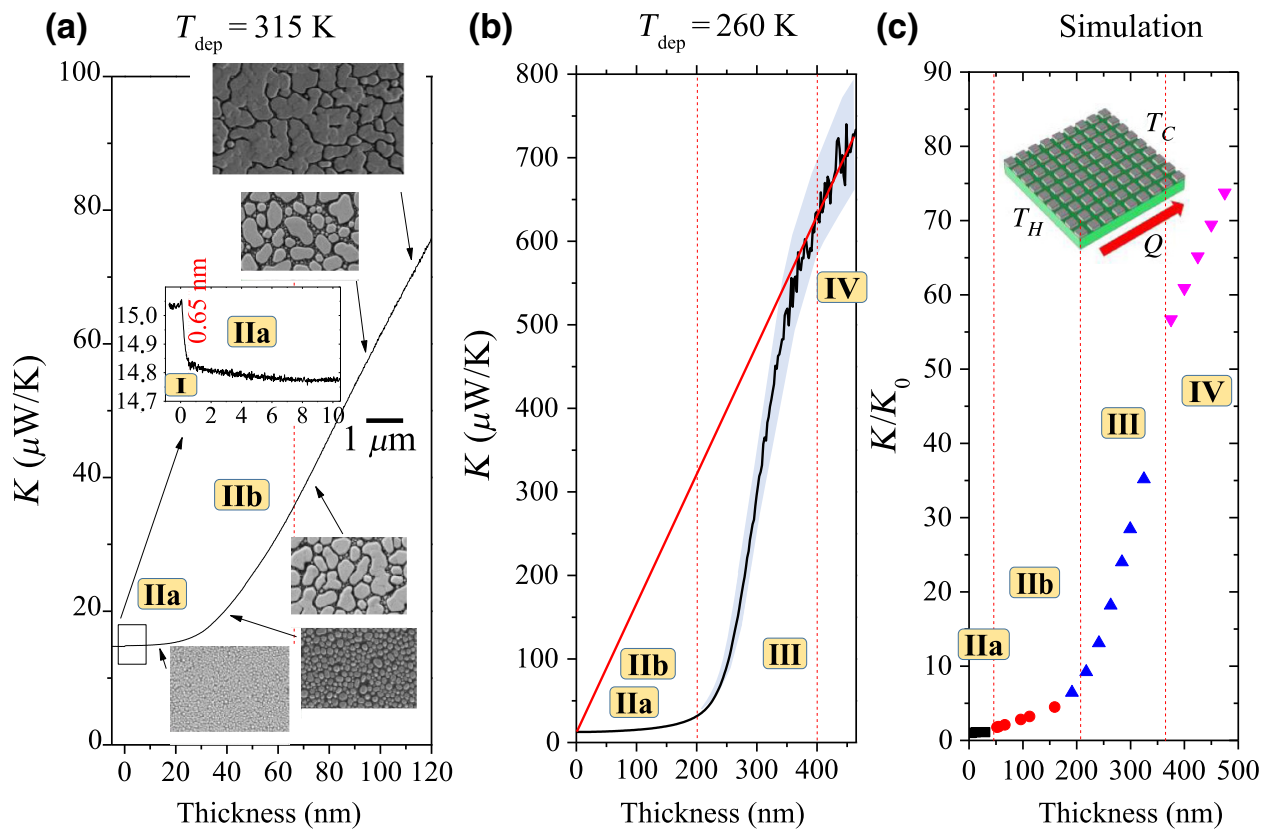

FIG. 6. (a) Thermal conductance versus thickness during deposition of In films: (a) $T_{\text {dep }}=315 \mathrm{~K}$. The electron microscopy images of films of varying nominal thickness show the microstructural evolution of the In layer. SEM images are acquired with a magnification of 100,000 , except the thickest one recorded at $50 \mathrm{k}$. The $1-\mu \mathrm{m}$ scale is provided inside the graph. The inset of (a) highlights the conductance drop during the early growth stages. (b) $T_{\text {dep }}=250 \mathrm{~K}$. Thickness range is extended to attain complete percolation and total coverage. The uncertainty of the thermal conductance is indicated by the blue shadow underneath the curve. (c) FEM simulation: normalized conductance versus In nominal thickness for representative structures with isolated islands of different sizes and percolated islands, finally forming a continuous layer. The different growth regimes are shown in roman letters. Inset of (c): Image of the 3D model, consisting of an array of $9 \times 9$ square In islands (gray) on a $\mathrm{SiN}_{x}$ thin membrane (green). 
The observed conductance drop of $1.5 \%$ is again an indication that phonons with mean free paths slightly larger than those typically accounted for in disordered solids are being scattered by the In/ $\mathrm{SiN}_{x}$ interface. Nevertheless, the minimum conductance found in this case cannot be correlated with a percolation threshold as we considered in TPD, since individual In islands are much more conductive than the TPD ones and they can contribute significantly to the thermal conduction even though they are still physically disconnected at this stage.

Interestingly, neither percolation threshold nor complete coverage of In grown on $\mathrm{SiN}_{x}$ are reached for a metallic layer as thick as $120 \mathrm{~nm}$ (nominal thickness), as clearly evidenced in the SEM images. As shown in Fig. 6(b), where growth characterization is performed up to $450 \mathrm{~nm}$, percolation starts to play a significant role at thicknesses around $200 \mathrm{~nm}$, where the conductance sharply increases due to continuous channel formation. Subsequently, the thermal conductance increase slows down, becoming linear with thickness. The corresponding fitting line [red line in Fig. 6(b)] is proportional to the thickness, meaning that the In layer is already continuous and the thermal conductivity can be evaluated from the slope of the conductance versus thickness. The value of $k$ for the continuous In layer deposited at $260 \mathrm{~K}$ is $47 \mathrm{~W} \mathrm{~m}^{-1} \mathrm{~K}^{-1}$, substantially lower than the tabulated value for In, $k=83 \mathrm{~W} \mathrm{~m}^{-1} \mathrm{~K}^{-1}$, probably due to smaller grain size and enhanced boundary scattering. From the images taken by SEM of the In layer at different thicknesses (insets of Fig. 6(a) and Fig. S5(b) in the Supplemental Material [27]), we approximate the coverage ratio and the mean island area.

As indicated in Figs. 6(a) and 6(b), we can differentiate four growth regimes: I: nucleation and initial small island growth, II: growth of islands, divided into IIa (with small isolated circular islands) and IIb (with larger islands irregularly shaped forming a bimodal island size distribution), III: island percolation forming continuous channels, and IV: vertical growth of a continuous film.

Since the conductance evolution is slightly different (region II is divided into two stages) than the one previously observed for TPD layers, we conduct FEM using the structural information provided by the electron microscopy images. We use a simplified representation of our sample by building an array of $9 \times 9$ In square islands on a $180-\mathrm{nm}$ thick $\mathrm{SiN}_{x}$ film. Changing the nominal thickness of the layer implies modulating the size and separation of the islands to match the island size and coverage ratio observed by SEM (see the Supplemental Material [27] for details on coverage calculations). The thermal conductance is monitored by imposing a heat flow and measuring the temperature difference arising on the simulated structure. The results of the simulation are shown in Fig. 6(c). The simulation closely predicts the increase of the slope of the curve $K_{2 \omega}\left(t_{\mathrm{smp}}\right)$ deposited at $315 \mathrm{~K}$ in a thickness range around $30-50 \mathrm{~nm}$. In this thickness range and up to the percolation threshold above $200 \mathrm{~nm}$, In islands are still isolated from one another and the increased conductance is due to the formation of larger islands as In evaporation proceeds. At around $200 \mathrm{~nm}$, the sudden increase in the slope is related to island percolation (region III starts). At the end of the percolation regime, a continuous film forms and the conductance is heavily dominated by the In film (region IV). Then, the conductance increases linearly with a slope given by the thermal conductivity of the film.

\section{CONCLUSIONS}

We develop a universal sensor with extreme accuracy and methodology able to perform real-time thermalconductance measurements during film growth. The technique is adaptive for any kind of material and thickness. Large sensitivity allows disentangling the evolution of film microstructure with thicknesses. By analyzing changes in the apparent thermal conductance versus thickness, differentiated growth stages can be identified, giving rise to a complete comprehension of the growth process for several materials. At early stages, the fast drop of the thermal conductance is related to nucleation and isolated clusters formation. This step is followed by a regime where clusters grow to form islands through coalescence and absorption of atoms or molecules from the gas phase, which dominates the variation of the heat conductance with thickness. In an intermediated stage, the percolation threshold is revealed by a conductance rise, while the final mode, where the thermal conductance changes linearly with thickness, corresponds to the formation and growth of a continuous film. In this regime, the thermal conductivity of the film can be directly derived from the slope of the conductance versus thickness plot.

The ad-hoc methodology presented here is easily extensible to devices with other substrate materials compatible with epitaxial growth. Using single crystalline membranes, the conductance reduction within the first stages of growth will be enhanced, increasing the sensitivity. The extreme sensitivity will pave the way to apply the technique to interesting phenomena such as phase changes during growth, size effects, or molecular orientation and density in organic glass films, among others.

\section{ACKNOWLEDGMENTS}

We acknowledge financial support from the Ministerio de Economía y Competitividad through Grants No. CSD2010-00044 (ConsoliderNANOTHERM), No. FIS2013-50304-EXP, and No. MAT2016-79579-R. P. Ferrando-Villalba and J. Ràfols-Ribé were in receipt of an FPU grant from the Spanish Ministry of Education, Culture and Sports. Ll. Abad was supported by the "Ramón y Cajal" program (Grant No. RyC-2013-12640) from Spanish Government. This work made use of the Spanish 
IMB-CNM-CSIC clean room (ICTS Network MICRONANOFABS).

P.F.V. drove most of the experiments, prepared the figures, and contributed to the writing of the main manuscript text. D.T. and Ll.A. designed and microfabricated the membrane-based sensors used in the experiments. J.R.R. carry out the experiments dealing with organic moleculesbased thin films. A.L. conceived the experiments and coordinated all the team. A.L., J.R.V., and G.G. wrote the main manuscript text. All authors reviewed the manuscript.

[1] J. A. Floro, E. Chason, and S. R. Lee, Real time measurement of epilayer strain using a simplified wafer curvature technique, MRS Proc. 406, 491 (1995).

[2] I. An, H. V. Nguyen, N. V. Nguyen, and R. W. Collins, Microstructural Evolution of Ultrathin Amorphous Silicon Films by Real-Time Spectroscopic Ellipsometry, Phys. Rev. Lett. 65, 2274 (1990).

[3] H. You, R. P. Chiarello, H. K. Kim, and K. G. Vandervoort, X-Ray Reflectivity and Scanning-Tunneling-Microscope Study of Kinetic Roughening of Sputter-Deposited Gold Films During Growth, Phys. Rev. Lett. 70, 2900 (1993).

[4] J. Dendooven, K. Devloo-Casier, M. Ide, K. Grandfield, M. Kurttepeli, K. F. Ludwig, S. Bals, P. Van Der Voort, and C. Detavernier, Atomic layer deposition-based tuning of the pore size in mesoporous thin films studied by in situ grazing incidence small angle X-ray scattering, Nanoscale 6, 14991 (2014).

[5] H. W. Yeom, T. Abukawa, M. Nakamura, S. Suzuki, S. Sato, K. Sakamoto, T. Sakamoto, and S. Kono, Initial stage growth of In and A1 on a single-domain $\mathrm{Si}(001) 2 \times 1$ surface, Surf. Sci. 341, 328 (1995).

[6] M. Schwartzkopf, A. Buffet, V. Körstgens, E. Metwalli, K. Schlage, G. Benecke, J. Perlich, M. Rawolle, A. Rothkirch, B. Heidmann, G. Herzog, P. Müller-Buschbaum, R. Röhlsberger, R. Gehrke, N. Stribeck, and S. V. Roth, From atoms to layers: in situ gold cluster growth kinetics during sputter deposition, Nanoscale 5, 5053 (2013).

[7] M. Schuisky, J. W. Elam, and S. M. George, In situ resistivity measurements during the atomic layer deposition of ZnO and W thin films, Appl. Phys. Lett. 81, 180 (2002).

[8] S. B. Arnason, S. P. Herschfield, and A. F. Hebard, Bad Metals Made with Good-Metal Components, Phys. Rev. Lett. 81, 3936 (1998).

[9] R. C. Dynes, J. P. Garno, and J. M. Rowell, TwoDimensional Electrical Conductivity in Quench-Condensed Metal Films, Phys. Rev. Lett. 40, 479 (1978).

[10] J. J. Colin, Y. Diot, P. Guerin, B. Lamongie, F. Berneau, A. Michel, C. Jaouen, and G. Abadias, A load-lock compatible system for in situ electrical resistivity measurements during thin film growth, Rev. Sci. Instrum. 87, 023902 (2016).

[11] Y. Pei, H. Wang, and G. J. Snyder, Band engineering of thermoelectric materials, Adv. Mater. 24, 6125 (2012).

[12] J. Kim, S. Lee, Y. M. Brovman, P. Kim, and W. Lee, Diameter-dependent thermoelectric figure of merit in single-crystalline Bi nanowires, Nanoscale 7, 5053 (2015).
[13] M. Hong, Z.-G. Chen, L. Yang, and J. Zou, Enhancing thermoelectric performance of $\mathrm{Bi}_{2} \mathrm{Te}_{3}$-based nanostructures through rational structure design, Nanoscale $\mathbf{8}, 8681$ (2016).

[14] D. G. Cahill, Analysis of heat flow in layered structures for time-domain thermoreflectance, Rev. Sci. Instrum. 75, 5119 (2004).

[15] M. Muñoz-Rojo, O. Caballero-Calero, A. F. Lopeandia, J. Rodriguez-Viejo, and M. Martin-Gonzalez, Review on measurement techniques of transport properties of nanowires, Nanoscale 5, 11526 (2013).

[16] L. Shi, D. Li, C. Yu, W. Jang, D. Kim, Z. Yao, P. Kim, and A. Majumdar, Measuring thermal and thermoelectric properties of one-dimensional nanostructures using a microfabricated device, J. Heat Transf. 125, 881 (2003).

[17] F. Völklein, Thermal conductivity and diffusivity of a thin film $\mathrm{SiO}_{2} / \mathrm{Si}_{3} \mathrm{~N}_{4}$ sandwich system, Thin Solid Films 188, 27 (1990).

[18] D. G. Cahill, Thermal conductivity measurement from 30 to $750 \mathrm{~K}$ : The $3 \omega$ method, Rev. Sci. Instrum. 61, 802 (1990).

[19] Y. K. Koh, S. L. Singer, W. Kim, J. M. O. Zide, H. Lu, D. G. Cahill, A. Majumdar, and A. C. Gossard, Comparison of the $3 \omega$ method and time-domain thermoreflectance for measurements of the cross-plane thermal conductivity of epitaxial semiconductors, J. Appl. Phys. 105, 054303 (2009).

[20] M. Muñoz Rojo, S. Grauby, J. M. Rampnoux, O. CaballeroCalero, M. Martin-Gonzalez, and S. Dilhaire, Fabrication of $\mathrm{Bi}_{2} \mathrm{Te}_{3}$ nanowire arrays and thermal conductivity measurement by $3 \omega$-scanning thermal microscopy, J. Appl. Phys. 113, 054308 (2013).

[21] S. Grauby, E. Puyoo, J.-M. Rampnoux, E. Rouvière, and S. Dilhaire, Si and SiGe nanowires: Fabrication process and thermal conductivity measurement by $3 \omega$-scanning thermal microscopy, J. Phys. Chem. C 117, 9025 (2013).

[22] A. Sikora, H. Ftouni, J. Richard, C. Hébert, D. Eon, F. Omnès, and O. Bourgeois, Highly sensitive thermal conductivity measurements of suspended membranes ( $\mathrm{SiN}$ and diamond) using a $3 \omega$-Völklein method, Rev. Sci. Instrum. 83, 054902 (2012).

[23] A. Sikora, H. Ftouni, J. Richard, C. Hébert, D. Eon, F. Omnès, and O. Bourgeois, Erratum: "Highly sensitive thermal conductivity measurements of suspended membranes (SiN and diamond) using a $3 \omega$-Völklein method" [Rev. Sci. Instrum. 83, 054902 (2012)], Rev. Sci. Instrum. 84, 029901 (2013).

[24] R. Sultan, A. D. Avery, J. M. Underwood, S. J. Mason, D. Bassett, and B. L. Zink, Heat transport by long mean free path vibrations in amorphous silicon nitride near room temperature, Phys. Rev. B 87, 214305 (2013).

[25] S.-M. Lee and D. G. Cahill, Heat transport in thin dielectric films, J. Appl. Phys. 81, 2590 (1997).

[26] F. Völklein and T. Starz, in XVI International Conference on Thermoelectrics (ICT'97). (Cat. No.97TH8291) (1997).

[27] See Supplemental Material at http://link.aps.org/supple mental/10.1103/PhysRevApplied.12.014007 for details: (I) on the microfabrication of the thermal sensor, (II) on the COMSOL model, (III) on the heat equation solution, (IV) on the electronics and data acquisition, (V) on the morphological characterization, AFM and SEM, of the TDP and In samples, (VI) thermal radiation test with a deposition at low 
temperatures, (VII) on the differential scanning calorimetry (DSC) of TPD deposited films and (VIII) the indium island growth model.

[28] R. Sultan, A. D. Avery, G. Stiehl, and B. L. Zink, Thermal conductivity of micromachined low-stress silicon-nitride beams from 77 to 325 K, J. Appl. Phys. 105, 043501 (2009).

[29] Y. Zhang, E. C. Glor, M. Li, T. Liu, K. Wahid, W. Zhang, R. A. Riggleman, and Z. Fakhraai, Long-range correlated dynamics in ultra-thin molecular glass films, J. Chem. Phys. 145, 114502 (2016).
[30] J. Ràfols-Ribé, R. Dettori, P. Ferrando-Villalba, M. Gonzalez-Silveira, L. Abad, A. F. Lopeandía, L. Colombo, and J. Rodríguez-Viejo, Evidence of thermal transport anisotropy in stable glasses of vapor deposited organic molecules, Phys. Rev. Mater. 2, 035603 (2018).

[31] S. S. Dalal, D. M. Walters, I. Lyubimov, J. J. de Pablo, and M. D. Ediger, Tunable molecular orientation and elevated thermal stability of vapor-deposited organic semiconductors, Proc. Natl. Acad. Sci. 112, 4227 (2015). 